УДК $546.59,544.773$

\title{
A Transformation of Intermediates of Sulfide Reduction of Gold(III) on the Pyrolytic Graphite
}

\author{
Alexander S. Romanchenko*, Maxim N. Likhatski, \\ Anton A. Karacharov and Yury L. Mikhlin \\ Institute of Chemistry and Chemical Technology SB RAS \\ FRC "Krasnoyarsk Science Center SB RAS" \\ 50/24 Akademgorodok, Krasnoyarsk, 660036, Russia
}

Received 26.02.2017, received in revised form 12.05.2017, accepted 18.07.2017

Solid products immobilized from colloids containing intermediates of the reduction of tetrachloroaurate-ions by sodium sulfide on the surface of highly oriented pyrolytic graphite were studied using scanning probe microscopy, cyclic voltammetry and X-ray photoelectron spectroscopy. An evaluation of surface topography and chemical state of both gold and sulfur taking place during colloid's pre-aging and under electrochemical oxidation in $0.001 \mathrm{M} \mathrm{HCl}$ solution were shown.

Keywords: electrochemistry, nanoparticles, gold, sols, immobilization, sulfides, surface, scanning probe microscopy, X-ray photoelectron spectroscopy.

DOI: $10.17516 / 1998-2836-0037$.

(C) Siberian Federal University. All rights reserved

* Corresponding author E-mail address: romaas82@mail.ru 


\title{
Трансформация продуктов
}

\section{сульфидного восстановления золота(III) \\ иммобилизованных на пиролитическом графите}

\author{
А.С. Романченко, М.Н. Лихацкий, \\ А.А. Карачаров, Ю.Л. Михлин \\ Институт химии и химической технологии СО РАН \\ ФИЦ «Красноярский научный иентр СО РАН» \\ Россия, 660036, Красноярск, Академгородок, 50/24
}

\begin{abstract}
Методами сканирующей зондовой микроскопии, ичиклической вольтамперометрии и рентгеновской фотоэлектронной спектроскопии (РФЭС) изучены иммобилизованные из гидрозолей продукты восстановления тетрахлораурат-ионов сульфидом натрия на поверхности высокоориентированного пиролитического графита. Показаны изменения характера покрытия и химического состояния золота и серы, происходящие в результате предварительного созревания золей и электрохимическом окислении в растворе 0,001 М соляной кислоть.
\end{abstract}

Ключевые слова: электрохимия, наночастицы, золото, золи, иммобилизация, сульфиды, поверхность, сканирующая зондовая микроскопия, рентгеновская фотоэлектронная спектроскопия.

\section{Введение}

Золотосодержащие нанокомпозиты находят применение в (фото)катализе, производстве сенсоров, медицине, тераностике, преобразовании солнечной энергии и т.д. Наночастицы золота, иммобилизованные на оксидных и углеродных подложках, уже несколько десятилетий используются в качестве низкотемпературных катализаторов окисления угарного газа [1], плазмонных фотокатализаторов для аэробного окисления органических веществ [2], компонентов электрокаталитического окисления воды [3] и пр. Большинство методов приготовления композитов, содержащих металлические наночастицы в качестве активных центров, основаны на применении заранее синтезированных частиц либо подразумевают восстановление металл-содержащего прекурсора на поверхности твердого носителя, в т.ч. после его пропитки [4]. Интермедиаты реакций восстановления металлов и их соединений из комплексов в растворе обладают адгезионными свойствами, отличными от свойствами наночастиц и ионных форм металлов, и могут найти применение в процессах приготовления катализаторов. Использование сравнительно долгоживущих промежуточных структур в отличие от традиционной пропитки исключает возможность восстановления ионов металлов материалом подложки, позволяет достичь высокой однородности пленки, замедляет коалесценцию частиц на поверхности носителя и т.д.

При взаимодействии сульфида натрия и тетрахлороаурикислоты в водном растворе в зависимости от условий (температуры, концентрации, в большей степени - молярного отношения 
реагентов $\left[\mathrm{Na}_{2} \mathrm{~S}\right]_{0} /[\mathrm{HAuCl}]_{0}$ ) могут образовываться либо металлические частицы с управляемыми свойствами [5-13], либо осадок аморфного сульфида золота (I), кристаллизации которого предшествует стадия относительно длительного (в течение часов и суток) существования пересыщенного раствора, содержащего, как нами было показано в серии работ [14-19], жидкие промежуточные структуры коллоидных размеров, природа которых остается малоизученной. Такие структуры проявляют ряд необычных свойств, в частности, в АСМ-эксперименте в жидкости (in situ) легко деформируются иглой микрокантилевера при “простукивании” (в полуконтактном режиме измерений) и таким образом не являются твердыми частицами, а представляют собой плотные капли [14]. Также продукты сульфидного восстановления обладают повышенной, по сравнению, например, с частицами цитратного гидрозоля золота, адгезией к поверхности высокоориентированного пирографита (ВОПГ) $[14,15]$, образуя на последнем плотную пленку, распадающуюся на воздухе на отдельные наночастицы размерами 2-5 нм. Разложение промежуточных структур наблюдали также под действием электронного пучка в условиях ПЭМ-эксперимента [15]. В нашей недавней работе [19] показано, что появление в растворе и укрупнение жидких промежуточных структур частично за счет роста, частично за счет коалесценции, являются необходимыми условиями нуклеации. Применение жидких преднуклеационных интермедиатов в качестве прекурсоров может открывать перспективы для получения инновационных наноматериалов, в том числе нанокомпозитов на основе наночастиц золота и/или сульфида золота на поверхности твердых носителей. Поведение жидких промежуточных структур, возникающих в реакции $\mathrm{HAuCl}_{4} \mathrm{c} \mathrm{Na} 2 \mathrm{~S}$, в условиях электродной поляризации и при старении на воздухе, слабо изучено, строение, состав и количественные характеристики сорбции таких интермедиатов на подложки разной природы в зависимости от различных условий и методов обработки практически не освещались в литературе. Целью настоящего исследования было изучение влияния электрохимического травления и старения золей на состав и свойства золотосодержащих пленок, осажденных на поверхности ВОПГ из растворов с жидкими промежуточными структурами, возникающими при взаимодействии $\mathrm{HAuCl}_{4}$ и сульфида натрия в водных растворах при фиксированном молярном отношении $\left[\mathrm{Na}_{2} \mathrm{~S}\right] /\left[\mathrm{HAuCl}_{4}\right]$ 3:1.

\section{Экспериментальная часть}

В работе использованы растворы золотохлористоводородной кислоты $\mathrm{HAuCl}_{4}$ (OAO «Красцветмет») и сульфида натрия, приготовленные из реактивов квалификации соответственно «хч» и «чда», и бидистиллированная вода. Типичная методика синтеза включала быстрое прибавление требуемого количества раствора 0,1 М сульфида натрия к 30 мл раствора $3,33 \cdot 10^{-4} \mathrm{M} \mathrm{HAuCl}_{4}$ и активное перемешивание раствора в течение, по меньшей мере, двух часов при комнатной температуре. Каплю реакционного раствора объемом 5-10 мкл наносили на свежеобновленную поверхность пирографита, давали ей высохнуть при комнатной температуре и осторожно промывали дистиллированной водой для удаления электролитов. Электрохимические исследования и окислительная обработка проводились на потенциостате P 30 SM фирмы Elins (г. Москва). Для проведения экспериментов капля исследуемого золя наносилась на поверхность кусочка пирографита (марка ZYB) и высушивалась при комнатной температуре. После высыхания капли поверхность промывалась от электролитов, затем к образцу подводился 
электрический контакт и погружался в раствор $0,01 \mathrm{M} \mathrm{HCl}$. После установления равновесия компромиссного потенциала (примерно 400 мВ относительно насыщенного хлорсеребряного электрода) производилась развертка потенциала со скоростью 5 мВ/с в анодном направлении. По достижению заданного потенциала электрод вынимался из раствора и высушивался на воздухе. Образцы исследовали методом атомно-силовой микроскопии (АСМ) в полуконтактной моде с помощью мультимодового сканирующего зондового микроскопа Solver P-47 (HT-МДТ, Москва) на воздухе при комнатной температуре. В АСМ зондом служил кремниевый кантилевер с типичной резонансной частотой около 150 кГц. Рентгеновские фотоэлектронные спектры записывали на спектрометре SPECS при возбуждении немонохроматизированным излучением $\mathrm{Mg} \mathrm{K \alpha}$ анода рентгеновской трубки при энергии пропускания 20 эВ для обзорных спектров или 8 эВ (узкие сканы) полусферического энергоанализатора PHOIBOS 150 MCD9. Разложение спектров выполняли с помощью программного пакета CasaXPS после вычитания нелинейного фона по Ширли с использованием гауссовско-лоренцевской формы максимумов. Отношения элементов на поверхности определяли по обзорным спектрам с учетом эмпирических коэффициентов чувствительности.

\section{Результаты и обсуждение}

На рис. 1 приведены типичные АСМ изображения, а на рис. 2 - рентгеновские фотоэлектронные спектры $\mathrm{Au} 4 \mathrm{f}$ и $\mathrm{S} 2 \mathrm{p}$ для образца, полученного при молярном отношении $\mathrm{Na}_{2} \mathrm{~S} / \mathrm{HAuCl}_{4}=3$, результаты разложения спектров на компоненты приведены в табл. 1 . АCM демонстрирует, что высохший золь представляет собой тонкую пленку или сетку из островков (рис. 1). Как было показано ранее [16], в данных условиях отсутствует металлическое золото, а продуктом является преимущественно аморфный сульфид золота. Однако на спектрах РФЭС представлены линии, характерные для металлического золота. Это можно объяснить тем, что нестабильный сульфид золота на поверхности разлагается в процессе подготовки образца с образованием металлического золота и серы, которая частично улетучивается в сверхвысоком вакууме $[15,16]$.

После старения золя в течение суток в растворе происходят изменения, в результате которых степень покрытия поверхности пирографита возрастает, причем наряду с разупорядоченным материалом образуется значительное число равномерно распределенных по поверхности ВОПГ сформировавшихся частиц золота либо агрегатов с латеральными размерами, достигающими порядка 300 нм (рис. 3). Спектры линий $\mathrm{Au} 4 \mathrm{f}$ и $\mathrm{S}$ 2p практически не изменяются, таким образом, в РФЭС золото также обнаруживается в металлической форме, а сера - в основном в дисульфидных $\mathrm{S}_{2}^{2-}$ и полисульфидных групп (рис. 2), но отношение $\mathrm{Au} / \mathrm{S}$ увеличивается и приближается к 2 , как в $\mathrm{Au}_{2} \mathrm{~S}$ (табл. 2). Данные по поверхностным концентрациям элементов и результаты разложения фотоэлектронных спектров для полученных образцов суммированы в табл. 1 и 2.

На рис. $1 \partial$ приведено типичное АСМ изображение частиц золя, состаренного в течение трех суток и осажденного на пирографите. В данном случае наблюдаются также пленка (монослой) из сформировавшихся частиц меньших размеров (до 200 нм) и весьма однородных. Как следует из АСМ изображений, частицы из состаренных золей лучше закрепляются на углеродной подложке. 

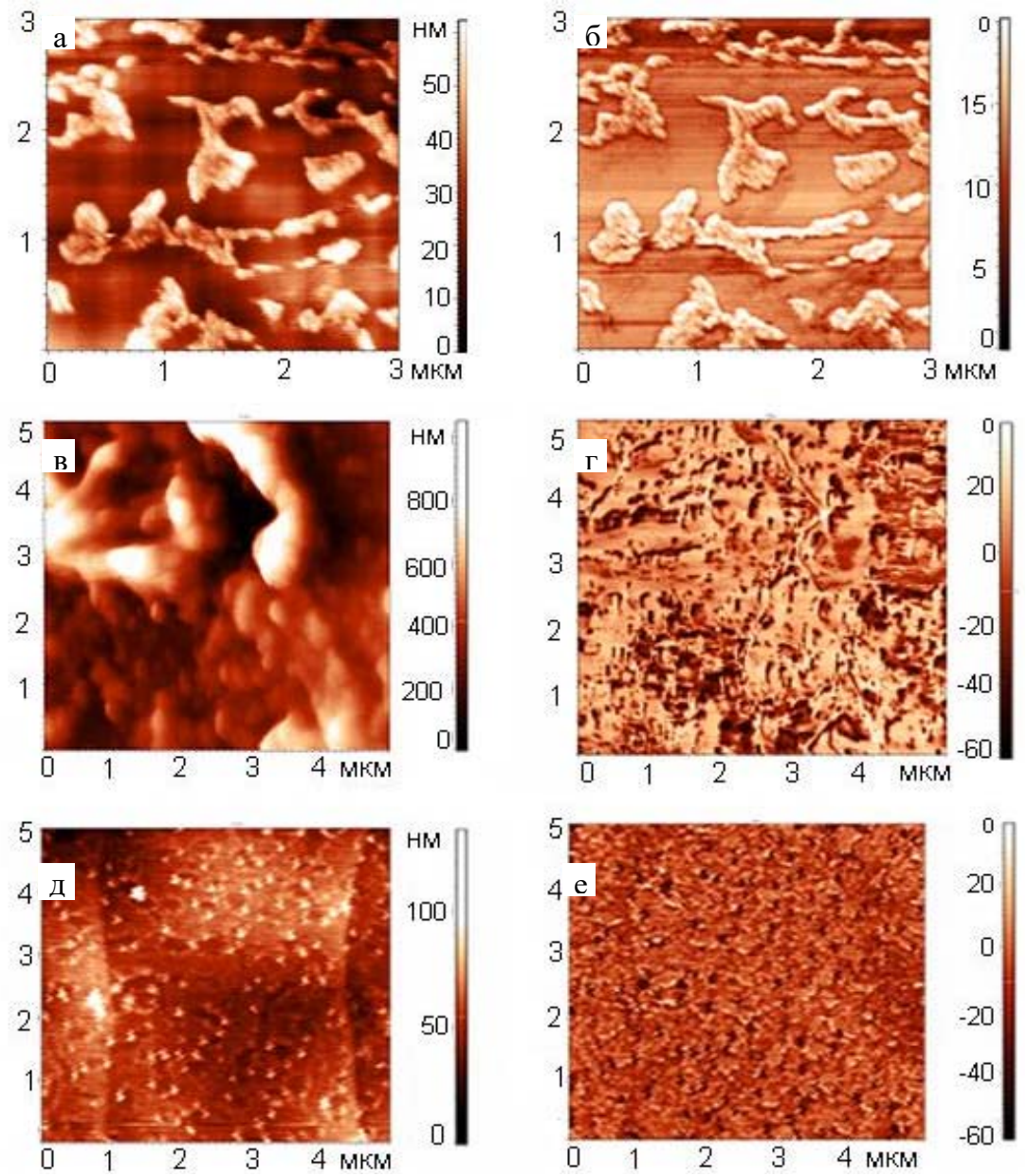

Рис. 1. Типичные АСМ-изображения (слева-рельеф, справа-фазовый контраст) поверхности пирографита с иммобилизованными продуктами сульфидного восстановления тетрахлороаурата, полученными при мольном отношении $\mathrm{S} / \mathrm{Au}=3,0: \mathrm{a}-$ исходный; в - состаренный за 1 сут.; д - состаренный 3 сут

Fig. 1. Typical AFM images (left panel - topography, right one - phase contrast) of pyrolytic graphite surface with immobilized products of sulfide reduction of tetrachloroauric acid obtained at S/Au molar ratio of 3.0: a) freshly prepared, b) aged for a day, c) aged for 3 days

На рис. 3 приведены типичные вольтамперные зависимости для золей и осадка, осажденных на пирографите, в растворе $0,01 \mathrm{M} \mathrm{HCl}$. Характерной общей особенностью в кислых растворах является наличие анодного максимума или плеча около 1 В (заметно возрастает после старения золя), плеча при 1,7 В и резкое возрастание анодного тока в области потенциала около 2 В. При окислении металлических Аu наночастиц, осажденных на пирографит, анодные токи ниже, а анодный максимум (плечо) сдвинут к более высоким потенциалам (около 1,2 В). Для того чтобы охарактеризовать превращения, происходящие в этих точках, электрод вынимался в нужной области потенциала из раствора и изучался методами АСМ и РФЭС.

После развертки потенциала до $1 \mathrm{~B}$ (начало появление анодного максимума) в растворе 0,01 M HCl исходные аморфные островки иммобилизованных продуктов образуют, по данным АCM, частицы с латеральными размерами порядка 80 нм и агломераты частиц размерами 

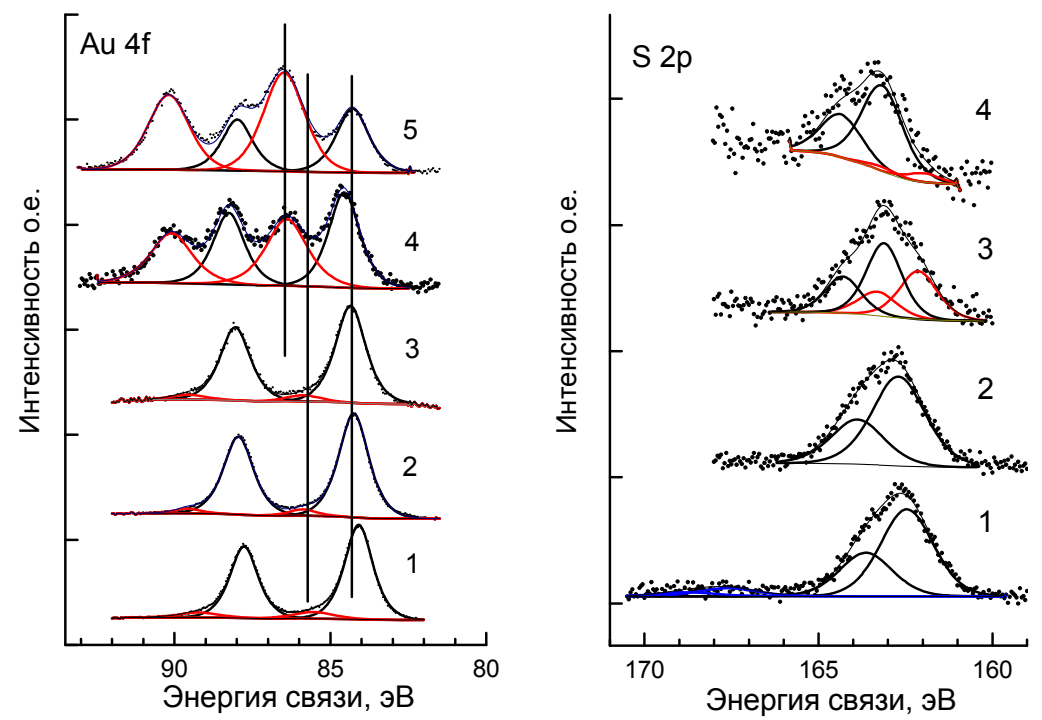

Рис. 2. Рентгеновские фотоэлектронные спектры линии $\mathrm{Au} 4 \mathrm{f}$ (a) и $\mathrm{S} \mathrm{2p}$ (б) частиц золя, полученного при мольном отношении $\mathrm{S} / \mathrm{Au}=3$, иммобилизованных на поверхность высокоориентированного пиролитического графита: 1 - свежий золь; 2 - состаренный в течение 1 суток и после развертки потенциала; 3 - до 1 В; 4 - до 1,7 В; 5 - до 2 В в растворе 0,01 M HCl, 5 мB/c

Fig. 2. 4f Au (a) and S 2p (b) X-ray photoelectron spectra of a sol obtained at S/Au molar ratio of 3.0 immobilized on the surface of highly oriented pyrolytic graphite: 1) freshly prepared sol, 2) the one aged for a day, and the ones after sweeping the electrode potential up to 3) $1 \mathrm{~V}$, 4) $1.7 \mathrm{~V}$, 5) $2 \mathrm{~V}$ in $0.01 \mathrm{M} \mathrm{HCl}$ solution, with sweep rate being $5 \mathrm{mV} / \mathrm{s}$
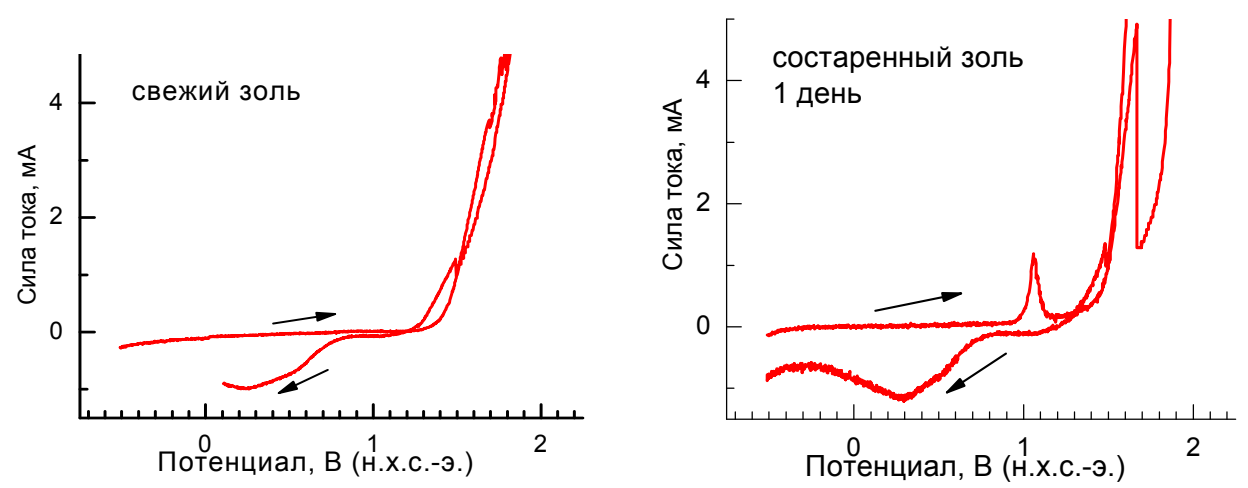

Рис. 3. Вольтамперные зависимости иммобилизованных на пирографите продуктов восстановления $\mathrm{HAuCl}_{4}$ сульфидом натрия, полученные в $0,01 \mathrm{M}$ растворе $\mathrm{HCl}$. Скорость развертки потенциала $5 \mathrm{mB} / \mathrm{c}$, $22{ }^{\circ} \mathrm{C}$

Fig. 3. Voltammetric curves acquired from the products of reduction of $\mathrm{HAuCl}_{4}$ with sodium sulfide immobilized on the surface of pyrolytic graphite in $0.01 \mathrm{M} \mathrm{HCl}$. The sweep rate was $5 \mathrm{mV} / \mathrm{s}$, the temperature was $22^{\circ} \mathrm{C}$

порядка 300 нм (рис. $4 a$, б). Спектры Au 4f не отличаются от образца до развёртки; а в спектрах линии $\mathrm{S} 2 \mathrm{p}$ появляются компоненты с низкой энергией связи - 162,1 эВ, что указывает на появление восстановленных форм серы - по-видимому, происходит разрыв связей между атомами серы и образование моносульфидных групп. Не исключено, что в данных условиях осуществляется окисление серы до более высоких степеней окисления -+4 и +6 , но ввиду вы- 
Таблица 1. Результаты разложения спектральных линий $\mathrm{Au} 4 \mathrm{f}$ и $\mathrm{S} 2 \mathrm{p}$ для частиц золя, полученного при мольном отношении $\mathrm{S} / \mathrm{Au}=3$, иммобилизованных на поверхность высокоориентированного пиролитического графита

Table 1. Results of deconvolution of $\mathrm{Au} 4 \mathrm{f}$ and S $2 \mathrm{p}$ bands acquired from the colloidal particles obtained at $\mathrm{S} / \mathrm{Au}$ molar ratio of 3.0 immobilized on the surface of highly oriented pyrolytic graphite

\begin{tabular}{|c|c|c|c|c|}
\hline \multirow[b]{2}{*}{ Образец } & \multicolumn{2}{|c|}{$\mathrm{Au} 4 \mathrm{f}_{7 / 2}$} & \multicolumn{2}{|c|}{$\mathrm{S} 2 \mathrm{p}_{3 / 2}$} \\
\hline & $\begin{array}{c}\text { Энергия связи } \\
\text { (эВ) }\end{array}$ & $\begin{array}{c}\text { Интенсивность } \\
(\%)\end{array}$ & $\begin{array}{c}\text { Энергия связи } \\
\text { (эВ) }\end{array}$ & $\begin{array}{c}\text { Интенсивность } \\
(\%)\end{array}$ \\
\hline Свежеприготовленный & $\begin{array}{l}84,1 \\
85,6\end{array}$ & $\begin{array}{c}91 \\
9\end{array}$ & $\begin{array}{l}162,5 \\
167,6\end{array}$ & $\begin{array}{c}91 \\
9\end{array}$ \\
\hline Состаренный за 1 сутки & $\begin{array}{l}84,3 \\
85,9 \\
\end{array}$ & $\begin{array}{c}95 \\
5 \\
\end{array}$ & 162,7 & 100 \\
\hline После развертки до 1.0 В & $\begin{array}{l}84,4 \\
85,9\end{array}$ & $\begin{array}{c}94 \\
6\end{array}$ & $\begin{array}{l}162,1 \\
163,1\end{array}$ & $\begin{array}{l}41 \\
60\end{array}$ \\
\hline После развертки до 1.7 В & $\begin{array}{l}84,6 \\
86,4\end{array}$ & $\begin{array}{l}52 \\
48 \\
\end{array}$ & $\begin{array}{l}162,0 \\
163,2\end{array}$ & $\begin{array}{c}7 \\
93\end{array}$ \\
\hline После развертки до $2.0 \mathrm{~B}$ & $\begin{array}{l}84,3 \\
86,5\end{array}$ & $\begin{array}{l}35 \\
65\end{array}$ & $\begin{array}{l}- \\
-\end{array}$ & $\begin{array}{l}- \\
-\end{array}$ \\
\hline
\end{tabular}

Таблица 2. Атомные поверхностные концентрации элементов для частиц золя, полученного при мольном отношении $\mathrm{S} / \mathrm{Au}=3$, иммобилизованных на поверхность высокоориентированного пиролитического графита, по данным РФЭС

Table 2. Surface atomic concentration of elements of colloidal particles obtained at S/Au molar ratio of 3.0 immobilized on the surface of highly oriented pyrolytic graphite as derived from XPS spectra

\begin{tabular}{|c|c|c|c|c|}
\hline Образец & $\mathrm{Au}$ & $\mathrm{Cl}$ & $\mathrm{S}$ & $\mathrm{C}$ \\
\hline Исходный & 2,7 & 0,3 & 3,2 & 93,8 \\
\hline Состаренный за 1 сутки & 2,6 & 1,9 & 1,7 & 93,8 \\
\hline После развертки до 1.0 В & 1,4 & 0,4 & 1,0 & 97,2 \\
\hline После развертки до 1.7 В & 0,8 & 3,6 & 0,6 & 96,0 \\
\hline После развертки до 2.0 В & 0,7 & 3,1 & 0,3 & 95,9 \\
\hline
\end{tabular}

сокой растворимости они полностью переходят в раствор и не регистрируются методом РФЭС. Количественный анализ элементов методом РФЭС показывает, что поверхностные концентрации золота и серы заметно уменьшаются по сравнению с исходным образцом. Таким образом, при развертке потенциала образца в анодном направлении в данных условиях происходит окисление серы, входящей в состав $\mathrm{Au}_{2} \mathrm{~S}$, что приводит к его разложению, при этом золото восстанавливается (ввиду неустойчивости $\mathrm{Au}_{2} \mathrm{~S}$ ) до металла и визуально наблюдается образование и укрупнение металлических наночастиц Аu. Сульфид-ионы, по-видимому, образуют адсорбированный слой на $\mathrm{Au}$, так как сера присутствует в РФЭС-спектрах практически всех образцов.

После развертки потенциала до 1,7 В в анодном направлении в растворе 0,01 $\mathrm{M} \mathrm{HCl,} \mathrm{как}$ можно видеть на АСМ-изображениях, отдельные частицы практически исчезают, агрегаты при этом остаются, а поверхность почти полностью покрыта рыхлым аморфным слоем золотосодер- 

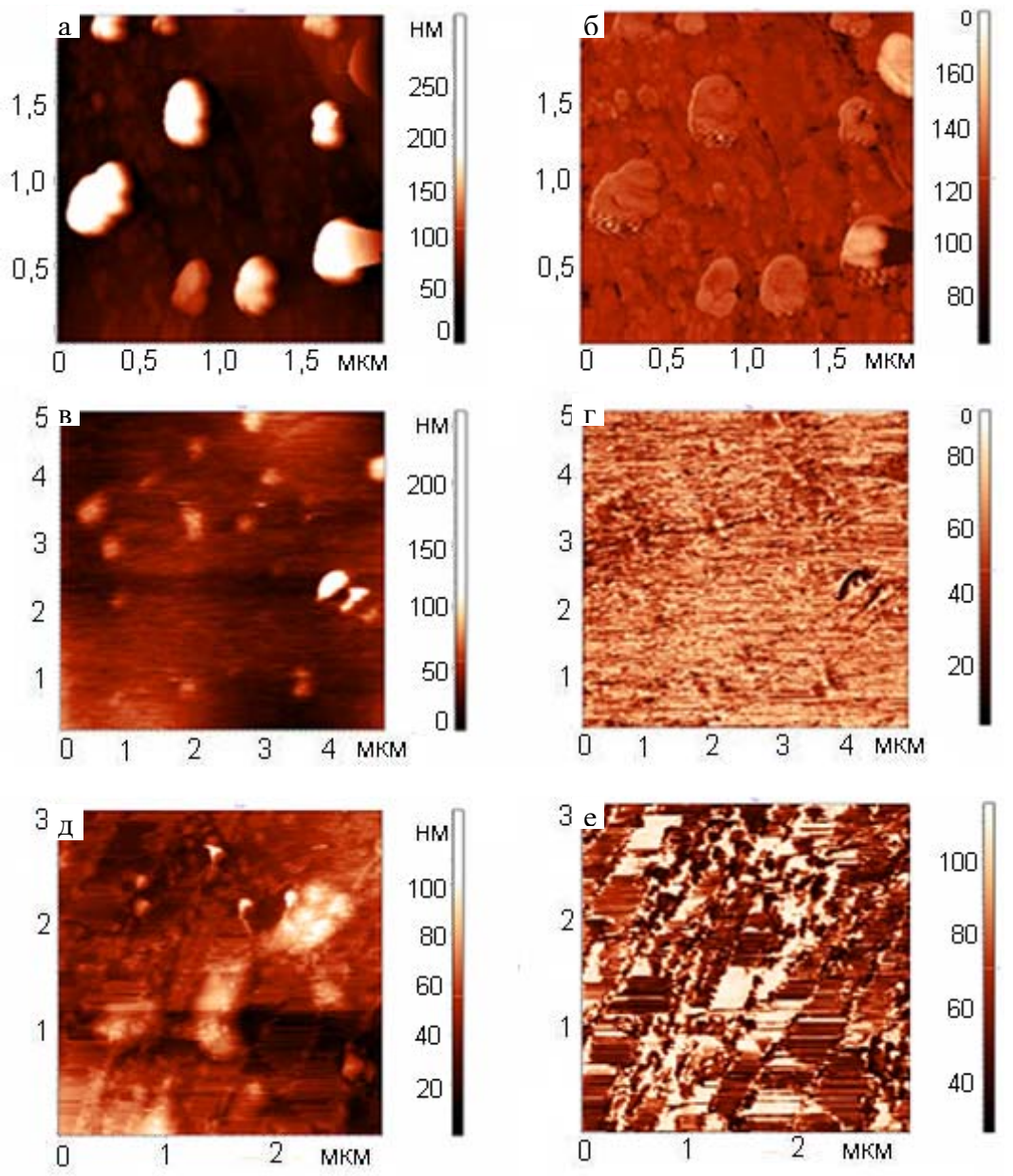

Рис. 4. Типичные АСМ-изображения рельефа (а, в, д) и фазового контраста (б, г, е) частиц золя, полученного при мольном отношении $\mathrm{S} / \mathrm{Au}=3$, иммобилизованных на поверхность пирографита, после развертки потенциала до $1 \mathrm{~B}(\mathrm{a}$, б), до 1,7 В (в, г) и до 2 В (д, е) в растворе $0,01 \mathrm{M} \mathrm{HCl}$

Fig. 4. Typical AFM images of topography (a, c, e) and phase contrast (b, d, f) of nanoparticles prepared at S/Au molar ratio of 3 immobilized on the surface of pyrolytic graphite after potential sweep up to $1 \mathrm{~V}(\mathrm{a}, \mathrm{b}), 1.7 \mathrm{~V}(\mathrm{c}$, d) and $2.0 \mathrm{~V}(\mathrm{e}, \mathrm{f})$ in $0.01 \mathrm{HCl}$

жащих продуктов (рис. 4в, г). Золото, по данным РФЭС, в значительной степени (практически 50 \%) переходит в состояние $\mathrm{Au}(\mathrm{III})$ - линия $\mathrm{Au} 4 \mathrm{f}$ имеет компоненту с энергией связи - 86,4 эВ (рис. 2). Сера полностью находится в полисульфидной форме $\mathrm{S}_{\mathrm{n}}{ }^{2-}$ (рис. 3б), ее концентрация на поверхности уменьшается, а концентрация хлора резко возрастает (табл. 2). Таким образом, большая часть атомов Аu теперь связана с хлорид-ионами в комплексные соединения Аu(III), хотя $\mathrm{Au}^{0}$ еще остается.

На рис. $4 \partial$, е представлено типичное АСМ-изображение поверхности пирографита с иммобилизованными продуктами восстановления золота после развертки потенциала в анодном направлении до 2 В. В данном случае наблюдаются редко расположенные частицы с поперечными размерами не более 100 нм (вероятно, остатки наблюдавшихся ранее агломератов) и также видна пленка из аморфных продуктов. РФЭС не обнаруживает серы 

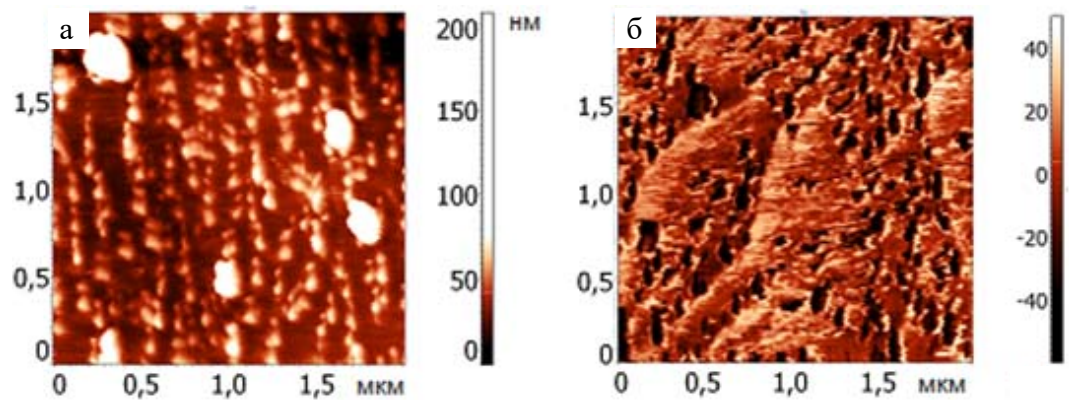

Рис. 5. АСМ-изображение частиц золя, полученного при мольном отношении $\mathrm{Na}_{2} \mathrm{~S} / \mathrm{HAuCl}_{4}=3,0$, иммобилизованных на поверхности пирографита после развертки потенциала до 2 В и затем к $-0,5$ В: a - рельеф поверхности; б - фазовый контраст

Fig. 5. AFM images of nanoparticles prepared at $\mathrm{Na}_{2} \mathrm{~S} / \mathrm{HAuCl} \mathrm{H}_{4}$ molar ratio immobilized on the surface of pyrolytic graphite after potential sweep to $2.0 \mathrm{~V}$, and then to $-0.5 \mathrm{~V}$ : a - topography, b-phase contrast

на поверхности, спектр $\mathrm{Au} 4 \mathrm{f}$ аналогичен спектру для случая развертки до 1,7 В (рис. 2, данные разложения в табл. 2), но только вклад компоненты $\mathrm{Au} 4 \mathrm{f}_{7 / 2}$ с энергией связи около 86,4 эВ заметно больше и составляет примерно $65 \%$. Следовательно, в составе продуктов преобладает комплекс золота (III) с хлорид-ионами, остальное золото (35 \%) представлено металлической формой.

Окисление при циклической развертке от компромиссного значения $(0,4 \mathrm{~B})$ до $2 \mathrm{~B}$, затем до - 0,5 В и к первоначальному значению, т.е. совершение полного цикла развертки, приводит к разрушению островковой или сетчатой структуры осажденных из золя продуктов и образованию однородных по размеру (около 40-80 нм, по данным АСМ) частиц золота, располагающихся ориентированными цепочками (рис. 5). В значительно меньших количествах наблюдаются также более крупные частицы или агрегаты (около 200 нм). Учитывая, что при высоких анодных потенциалах золото практически удаляется с поверхности, а при прохождении развертки в катодном направлении снова обнаруживается на поверхности, в данном случае наблюдается его восстановление до $\mathrm{Au}(0)$.

\section{Обсуждение результатов}

Основным золотосодержащим продуктом восстановления тетрахлороаурат-иона, полученным при отношении начальных концентраций реагентов $\left[\mathrm{Na}_{2} \mathrm{~S}\right]_{0} /\left[\mathrm{HAuCl}_{4}\right]_{0} 3: 1$ в кислой среде $(\mathrm{pH} \approx 2,0)$ при комнатной температуре после осаждения на углеродный носитель либо в составе выделенного и промытого осадка, согласно ранее полученным нами результатам моделирования EXAFS-спектров [16], является аморфный сульфид золота (I). Тонкий поверхностный слой полученного таким образом материала уже на воздухе и в большей степени - в сверхвысоком вакууме, а также под действием рентгеновского либо электронного пучка разлагается с образованием наночастиц золота и покрытием ди- и полисульфидного типа. Наблюдаемые на изображениях АСМ при старении на воздухе либо при поляризации осажденного продукта при умеренных потенциалах ( $\approx 1 \mathrm{~B})$ изменения пленки, по всей видимости, связаны с окислением под действием кислорода воздуха либо, соответственно, электроокисления, ди- и полисульфидного адслоя на поверхности наночастиц, который, вероятно, обеспечивает электростатическую ста- 
билизацию исходных частиц. Дальнейшее увеличение электродного потенциала инициирует полуреакции окисления сульфида золота с частичным переходом в раствор с учетом состава солевого фона, хлорокомплексов трехвалентного $\left[\mathrm{AuCl}_{4}\right]^{-}$и одновалентного золота $\left[\mathrm{AuCl}_{2}\right]$. Видимая на изображениях АCM (рис. 6в-е) пленка, экранирующая частицы от «простукивания» иглой микрокантилевера, возникает, по-видимому, как следствие переосаждения продуктов окисления сульфида золота (I). Избирательный характер электрорастворения наночастиц, некоторое упорядочение вдоль ступеней поверхности пирографита, обнаруживаемое на изображениях АCM (рис. 7), определяются, на наш взгляд, стабилизирующим взаимодействием поверхностных атомов наночастиц и/или лигандов, покрывающих частицы, с функциональными группами поверхности пирографита [20], концентрация которых здесь должна быть выше по сравнению с таковой на террасах.

\section{Заключение}

В работе показано, что имеет место трансформация промежуточных продуктов восстановления тетрахлороаурикислоты сульфидом натрия при старении гидрозоля на воздухе, которая приводит к формированию при последующем осаждении продуктов на поверхность пирографита оформленных наночастиц и агрегатов в отличие от свежеприготовленных форм Au-S, образующих при осаждении сплошную аморфную пленку. Электрохимическое окисление при умеренных потенциалах (до 1 B н.х.с.э.) влечет за собой к разложение $\mathrm{Au}_{2} \mathrm{~S}$, входящего в состав пленки и формированию наночастиц металлического золота. При высоких окислительных потенциалах происходит окисление золота до трехвалентного состояния и образование хлоридных комплексов, которые постепенно переходят в раствор. Процесс удаления золота с поверхности пирографита обратим - при наложении катодного потенциала $\mathrm{Au}(\mathrm{III})$ востонавливается до металла с образованием наночастиц, распределение которых на поверхности имеет упорядоченный характер. Таким образом, путем воздействия электрохимического потенциала на иммобилизованные на углеродной поверхности интермедиаты возможно получать металлические наночастицы с заданными размерами и степенью покрытия.

\section{Благодарности}

Исследование выполнено при финансовой поддержке Российского фонда фундаментальных исследований, Правительства Красноярского края, Красноярского краевого фонда поддержки научной и научно-технической деятельности в рамках научного проекта № 17-45240759 p_a.

\section{Список литературы}

1. Taktoshi A., Haruta M. Size- and structure-specificity in catalysis by gold clusters. Chem. Lett., 2014. Vol. 43. P. 380-387.

2. Clavero C. Plasmon-induced hot-electron generation at nanoparticle/metal-oxide interfaces for photovoltaic and photocatalytic devices. Nature Photonics, 2014. Vol. 8. P. 95-103.

3. Kuo Ch.-H., Weikun Li, Pahalagedara L., El-Sawy A.M., Kriz D., Genz N., Giuld G., Ressler Th., Suib S.L., He J. Understanding the role of gold nanoparticles in enhancing the catalytic activity of manganese oxides in water oxidation reaction. Angew. Chem., 2014. Vol. 126. P. 1-7.

$$
-425-
$$


4. Крылов О.В. Гетерогенный катализ. М.: ИКЦ «Академкнига», 2004. 679 с. [Krylov O.V. Heterogeneous catalysis. M.: PBC “Akademkniga”, 2004. 679 p. (in Russ.)].

5. Zhou H.S., Honma I., Komiyama H., Haus J.W. Controlled synthesis and quantum-size effect in gold-coated nanoparticles. Phys. Rev. B., 1994. Vol. 50, P. 12052-12057.

6. Averitt R.D., Sarkar D., Halas N.J. Plasmon resonance shifts of Au-coated $\mathrm{Au}_{2} \mathrm{~S}$ nanoshells: insight into multicomponent nanoparticle growth. Phys. Rev. Lett., 1997. Vol. 78, P. 4217-4220.

7. Halas N.J., West J.L. Nanoshell-mediated near-infrared thermal therapy of tumors under magnetic resonance guidance. Proc. Nat. Acad. Sci., 2002. Vol. 100, P. 13549-13554.

8. Norman T.J.Jr., Grant C.D., Magana D., Zhang J.Z., Liu J., Cao D., Bridges F., Van Buuren A. Near Infrared Optical Absorption of Gold Nanoparticle Aggregates. J. Phys. Chem. B, 2002. Vol. 106. P. 7005-7012.

9. Schwartzberg A.M., Grant C.D., Wolcott A., Talley C.E., Huser T.R., Bogomolni R., Zhang J.Z. Unique gold nanoparticle aggregates as a highly active surface-enhanced Raman scattering substrate. J. Phys. Chem. B, 2004. Vol. 108, P. 19191-19197.

10. Zhang J.Z., Schwartzberg A.M., Norman T. Jr., Grant C.D., Liu J., Bridges F., van Buuren T. Comment on "Gold Nanoshells Improve Single Nanoparticle Molecular Sensors". Nano Lett., 2005. Vol. 5, P. 809-810.

11. Hirsch L.R., Stafford R.J., Bankson J.A., Sershen S.R., Rivera B., Price R.E., Hazle J.D., Schwartzberg A.M., Grant C.D., van Buuren T., Zhang J.Z. Reduction of $\mathrm{HAuCl}_{4}$ by $\mathrm{Na}_{2} \mathrm{~S}$ Revisited: The Case for $\mathrm{Au}$ Nanoparticle Aggregates and Against $\mathrm{Au}_{2} \mathrm{~S} / \mathrm{Au}$ Core/Shell Particles. J. Phys. Chem. C, 2007. Vol. 111, P. 8892-8901.

12. Diao J.J., Hao C. Near infrared surface plasmon resonance of gold tabular nanostructures in the $\mathrm{HAuCl}_{4}-\mathrm{Na}_{2} \mathrm{~S}$ reaction. J. Chem. Phys., 2006. Vol. 124, P. 116103.

13. Geng J., Thomas M.D.R., Shephard D.S., Johnson B.F.G. Suppressed electron hopping in a Au nanoparticle $/ \mathrm{H}_{2} \mathrm{~S}$ system: development towards a $\mathrm{H}_{2} \mathrm{~S}$ nanosensor. Chem. Commun., 2005. Vol. 14, P. 1895-1897.

14. Лихацкий М.Н., Михлин Ю.Л. Влияние сульфид-ионов на образование и свойства наночастиц золота в водных растворах. Физика и химия стекла, 2007. Т. 33 (4), С. 580 584. [Likhatski M.N., Mikhlin Y.L. Influence of sulfide ions on the formation and properties of gold nanoparticles in aqueous solutions. Glass Physics and Chemistry, 2007. Vol. 33(4). P. 580-584. (in Russ.)].

15. Mikhlin Y., Likhatski M., Karacharov A., Zaikovski Vol., Krylov A. Formation of gold and gold sulfide nanoparticles and mesoscale intermediate structures in the reactions of aqueous $\mathrm{HAuCl}_{4}$ with sulfide and citrate ions Phys. Chem. Chem. Phys., 2009. Vol. 11, P. 5445-5454.

16. Mikhlin Yu., Tomashevich Ye., Likhatski M., Romanchenko A., Erenburg S., Trubina S. XAS and XPS examination of the Au-S nanostructures produced via the reduction of aqueous gold (III) by sulfide ions Journal of Electron Spectroscopy and Related Phenomena, 2010. Vol. 177, P. 24-29.

17. Mikhlin Yu.L., Romanchenko A.S., Likhatski M.N., Karacharov A.A., Erenburg S.B., Trubina S.Vol. Understanding the initial stages of precious metals precipitation: nanoscale metallic and sulfidic species of gold and silver on pyrite surfaces. Ore Geol. Rev., 2011. Vol. 42. P. 47-64. 
18. Mikhlin Yu.L., Karacharov A.A., Likhatski M., Podlipskaya T., Zizak I. Direct observation of liquid pre-crystallization intermediates during the reduction of aqueous tetrachloroaurate by sulfide ions. Physical Chemistry Chemical Physics, 2014. Vol. 16. P. 4538 - 4543.

19. Likhatski M., Karacharov A., Kondrasenko A., Mikhlin Yu. On a role of liquid intermediates in nucleation of gold sulfide nanoparticles in aqueous media. Faraday Discussions, 2015. Vol. 144. P. 235-245.

20. Hyun-Suk Choo, Taro Kinumoto, Masafumi Nose, Kohei Miyazaki, Takeshi Abe, Zempachi Ogumi Electrochemical oxidation of highly oriented pyrolytic graphite during potential cycling in sulfuric acid solution. Journal of Power Sources, 2008. Vol. 185. P. 740-746. 